Revue internationale P.M.E.

Économie et gestion de la petite et moyenne entreprise

\title{
Partage d'information et performance de coentreprises implantées par les PME québécoises dans les pays en développement
}

\section{Claude Marcotte et Pierre-André Julien}

Volume 8, numéro 2, 1995

URI : https://id.erudit.org/iderudit/1008354ar

DOI : https://doi.org/10.7202/1008354ar

Aller au sommaire du numéro

Éditeur(s)

Presses de l’Université du Québec

ISSN

0776-5436 (imprimé)

1918-9699 (numérique)

Découvrir la revue

Citer cette note

Marcotte, C. \& Julien, P.-A. (1995). Partage d'information et performance de coentreprises implantées par les PME québécoises dans les pays en développement. Revue internationale P.M.E., 8(2), 175-201.

https://doi.org/10.7202/1008354ar
Résumé de l'article

Deux hypothèses de recherche ont été examinées dans cette étude. La première établit une relation entre le partage d'information entre les partenaires d'une coentreprise, le mode de contrôle managérial utilisé, les coûts de transaction, les effets de synergie et la performance. La seconde hypothèse suggère que les PME diffèrent des grandes entreprises quant à la forme de contrôle managérial qu'elles privilégient dans les coentreprises. À l'aide d'une étude de cas effectuée auprès de sept coentreprises établies en Afrique, nous avons pu constater que la première hypothèse était partiellement confirmée alors que la deuxième l'était entièrement. Ces résultats qualitatifs devraient cependant être corroborés par d'autres études qui réuniraient un plus grand nombre de coentreprises et intégreraient certaines variables environnementales et structurelles. 


\title{
Partage d'information et performance de coentreprises implantées par les PME québécoises dans les pays en développement
}

\author{
Claude MARCOTTE* \\ Pierre-André JULIEN** \\ Université du Québec à Trois-Rivières
}

MOTS CLÉS

Partage d'information - Coentreprises - Contrôle managérial

Coûts de transaction - Synergie - Performance

\begin{abstract}
RÉSUMÉ
Deux hypothèses de recherche ont été examinées dans cette étude. La première établit une relation entre le partage d'information entre les partenaires d'une coentreprise, le mode de contrôle managérial utilisé, les coûts de transaction, les effets de synergie et la performance. La seconde hypothèse suggère que les PME diffèrent des grandes entreprises quant à la forme de contrôle managérial qu'elles privilégient dans les coentreprises. À l'aide d'une étude de cas effectuée auprès de sept coentreprises établies en Afrique, nous avons pu constater que la première hypothèse était partiellement confirmée alors que la deuxième l'était entièrement. Ces résultats qualitatifs devraient cependant être corroborés par d'autres études qui réuniraient un plus grand nombre de coentreprises et intégreraient certaines variables environnementales et structurelles.
\end{abstract}

* Claude Marcotte possède une maîtrise en psychologie de l'Université d'Ottawa et une maîtrise en gestion des PME de l'Université du Québec à Trois-Rivières. Il est présentement aux études pour l'obtention du doctorat en administration de l'Université du Québec à Montréal. Son sujet de spécialisation est «Les alliances technologiques entre PME ». Il a déjà publié trois livres en psychologie et quatre articles portant sur les problèmes d'apprentissage.

** Pierre-André Julien est docteur en sciences économiques de l'Université Catholique de Louvain (Belgique). Il est aussi diplômé en science administrative de l'Université Laval. Il est titulaire de la Chaire Bombardier Sea-Doo / Ski-Doo en gestion du changement technologique dans les PME à l'Université du Québec à Trois-Rivières et directeur de la Revue Internationale PME. Il est présentement consultant auprès de l'Organisation de coopération et de développement économique (OCDE) à Paris.

Adresse: Chaire Bombardier, Université du Québec à Trois-Rivières, C.P. 500, Trois-Rivières (Québec) G9A 5H7. 


\begin{abstract}
Two research hypotheses were examined in this study. First, it was hypothesized that there is a relationship between the following variables: the information sharing process between the joint venture's partners, the managerial control mode, the transaction costs, the synergy effects and performance. Secondly, it was hypothesized that small and medium-sized firms would differ from larger enterprises in the use of managerial control modes. We did a case study on seven joint ventures operating in Africa. The case study data suggest that the first hypothesis is partially confirmed, and that the second one is confirmed in these particular instances. Those qualitative data will have to be verified with a larger number of firms and through the inclusion of other variables dealing with the environment and the structure of the firms.
\end{abstract}

\title{
RESUMEN
}

Dos hipótesis de investigación han sido examinadas en éste estudio. La primera establece una relación entre la comunicación de las informaciónes existentes entre los asociados de una co-empresa, el modo utilizado del control de la gestion, los costos de transacción, los efectos de la sinergía y el rendimiento. La segunda hipótesis sugiere que las PME se diferencian de las grandes empresas respecto a la forma de control de gestion que allas favorecen en las co-empresas. Basaudose en el estudio de casos efectuados en siete co-empresas establicidas en Africa. Nosotros hemos podido constator que la primera hipótesis estaba parcialmente confirmada mientras que la segunda hipótesis estaba completamente confirmada. Estos resultados calitativos deberían sin embargo ser corroborados por otros estudios que reunirían un mayor número de co-empresas y quienes integrarian ciertas variables estructurales $y$ del medio ambiente.

\section{Introduction}

Même s'il est difficile d'obtenir des données statistiques précises sur le phénomène des alliances stratégiques internationales, il semble que le nombre de ces alliances ait augmenté sensiblement depuis le milieu des années 1970 (Morris et Hergert, 1987). Une des formes d'alliances de plus en plus utilisée par les grandes entreprises désireuses d'exporter vers les pays en développement est la coentreprise : une grande firme ou quelques grandes firmes opérant dans des pays industrialisés s'associent avec une ou des firmes établies dans un pays en développement ; une nouvelle entité autonome, la coentreprise, est alors implantée dans ce pays.

Cette tendance ne se limite pas aux grandes entreprises. Le nombre d'ententes de coentreprises conclues entre PME de pays industrialisés et PME de pays en développement semble avoir augmenté depuis une vingtaine d'années, même si le mouvement semble plus prononcé dans les grandes entreprises (Herbolzheimer et Ouane, 1985). Les PME des pays industrialisés 
sont de plus en plus encouragées par les agences de développement international à créer des partenariats d'affaires avec d'autres entreprises de pays en développement.

Un des avantages potentiels de ces partenariats pour une PME québécoise serait l'acquisition d'information sur le pays visé. Les informations d'affaires sont souvent difficiles à obtenir sur ces pays, en partie à cause du manque d'infrastructures pouvant permettre une collecte fiable et une diffusion appropriée de l'information. De plus, les particularités d'ordre culturel, légal et politique de ces pays peuvent faire en sorte que les autres méthodes d'exportation se révèlent difficiles à utiliser, surtout pour les PME qui ne disposent habituellement que de peu de ressources pour l'investissement à l'étranger.

En choisissant un partenaire qui connaît bien l'environnement général du pays visé et en partageant avec ce partenaire les informations nécessaires à la bonne marche de la coentreprise, ainsi que certaines ressources financières, humaines et techniques, une PME devrait théoriquement mieux réussir son implantation à l'étranger.

Mais, dans les faits, y a-t-il une relation entre le partage de l'information entre les partenaires et la performance des coentreprises? Et si cette relation existe, s'agit-il d'un lien direct entre ces deux variables, ou bien est-ce que le partage d'information agit d'abord sur d'autres variables qui, à leur tour, influencent la performance?

C'est ce que cette étude veut vérifier à partir d'une étude de cas. L'interrelation entre les variables suivantes sera examinée: le partage d'information entre les partenaires d'une coentreprise, le mode de contrôle managérial utilisé, les coûts de transaction, les effets de synergie et la performance. Pour ce faire, nous traiterons dans un premier temps de la problématique sousjacente à cette étude. Ensuite, nous présenterons la méthodologie utilisée et terminerons par la présentation des résultats et les conclusions.

\section{Problématique}

\subsection{L'acquisition d'information comme un des avantages des coentreprises}

Qu'est-ce qui peut motiver des dirigeants d'entreprises à former des coentreprises? Les inconvénients et les risques inhérents à cette forme d'organisation sont souvent cités dans la littérature: problèmes de communication et de coordination avec les partenaires, risques de comportements opportunistes de la part d'un partenaire qui peut devenir un concurrent redoutable une fois 
l'expertise acquise. Les avantages que perçoivent les responsables d'entreprises doivent donc compenser les inconvénients et les risques courus.

La liste des motivations possibles que l'on retrouve dans la littérature inclut les éléments suivants (Vernon et Wells, 1986; Contractor et Lorange, 1988):

1. Motivations politiques: surtout la nécessité de satisfaire les exigences gouvernementales du pays où l'on veut investir, en ce qui concerne la propriété des entreprises locales. Même dans les pays où ces exigences sont abolies, la présence d'un partenaire local peut constituer un atout politique important (contacts plus faciles avec la bureaucratie locale).

2. Motivations économiques et financières

- les économies d'échelle par la mise en commun des actifs ;

- la diminution des coûts d'investissement et des risques financiers.

3. Motivations d'ordre concurrentiel: avantages concurrentiels accrus, résultant de parts de marché plus grandes ou encore d'une stratégie visant à bloquer l'avance d'un concurrent commun.

4. Motivations d'ordre informationel: acquisition d'information sur l'environnement général du pays visé ; acquisition de savoir-faire, de technologie.

Ces considérations théoriques nous amènent à réaliser que les firmes peuvent tenir compte, dans leurs décisions d'investissement, non seulement des coûts et des bénéfices tangibles ou financiers, mais aussi des coûts et des bénéfices intangibles reliés particulièrement à l'échange d'information, de savoir-faire et de technologie. De même, l'évaluation que font les partenaires de la performance de leur coentreprise peut inclure d'autres éléments que des considérations strictement économiques ou financières, comme le font d'ailleurs remarquer Geringer et Hébert (1989 et 1991). L'acquisition d'information auprès du partenaire pourrait être une des variables importantes en relation avec la performance des coentreprises, pour autant que cette performance soit évaluée «de l'intérieur », c'est-à-dire par chacun des partenaires selon ses propres critères et non à partir de critères « objectifs » prédéfinis par le chercheur.

\subsection{Partage d'information et performance des coentreprises}

La thèse de doctorat de Beamish (1984) a fourni les principales données empiriques indiquant une relation entre le partage d'information entre les partenaires et la performance des coentreprises. Les résultats de cette enquête 
tendaient à démontrer que les responsables d'entreprises qui se disaient satisfaits de la performance de leur coentreprise étaient ceux qui recherchaient et obtenaient des informations spécifiques de leurs partenaires. Les partenaires des pays industrialisés obtenaient surtout des informations utiles sur l'environnement socio-économique du pays hôte, tandis que les partenaires du pays en développement obtenaient des informations reliées à des connaissances technologiques plus poussées. À l'inverse, les coentreprises évaluées comme non performantes étaient celles où les répondants ne recherchaient pas une contribution du partenaire quant à l'information à recueillir.

Cette étude de Beamish a le mérite d'offrir des données empiriques dans un champ d'étude encore trop théorique. Cependant, certaines limites méthodologiques et conceptuelles rendent difficile l'interprétation de ces résultats. Beamish infère, dans son étude, une relation de causalité entre les besoins d'information des partenaires et la performance des coentreprises, ce qu'une approche descriptive comme celle qu'il a utilisée ne permet pas. On peut également se demander si, outre l'aspect du contenu et de l'importance des informations recherchées, le processus d'interaction entre les partenaires quant à l'échange d'information ne constitue pas une deuxième dimension informationnelle importante. Dans ce processus d'échange d'information entre les partenaires, les aspects de comportements sociaux et culturels des partenaires, de même que ceux de l'environnement où évoluent ces partenaires, peuvent s'ajouter aux variables identifiées par Beamish. L'étude de cette relation nécessite peut-être l'utilisation de méthodes qui vont plus en profondeur que ce que permet l'enquête par questionnaire utilisée par Beamish. Ce qui explique pourquoi quelques auteurs, en se basant sur l'expérience vécue dans les PME, ont fait diverses observations pouvant nous aider à isoler certains éléments du processus d'échange d'information et montrant que la plupart des PME des pays en développement se comportent différemment des PME des pays industrialisés.

Le premier aspect des différences est celui du temps ou du synchronisme des échanges. Par exemple, Delalande (1989) rapporte que des coentreprises dans les pays en développement ont connu des crises graves parce que l'information d'un des partenaires fut trop tardive. La différence de conception du temps entre la plupart des pays industrialisés et les pays en développement a été constatée à maintes reprises (Austin, 1990; Harris et Moran, 1987). Les informations seraient également plus lentes à circuler à travers les différents paliers de l'organisation dans les pays en développement.

Un degré moindre d'ouverture face à l'échange d'information avec d'autres firmes est également rapporté. El Alami (1987) rapporte qu'en Afrique «l'association en affaires n'est pas bien vécue tellement les relations des associés entre eux et leurs relations avec l'entreprise sont personnalisées ». 
Cela oblige l'entrepreneur occidental à se montrer «diplomate» dans sa façon d'acquérir des informations et aussi d'en donner: il doit éviter de paraître arrogant.

Un troisième aspect concerne le degré d'opportunisme ou d'honnêteté dans les échanges. Certains auteurs, comme Guéneau (1986), soulignent qu'en dehors des petites collectivités que sont la famille au sens très large ou le clan, la fiabilité des informations et des comportements est difficile à obtenir, du moins lors des premiers contacts. Bien entendu, les comportements opportunistes constituent un risque peu importe le pays où l'on investit. Cependant, la forme que prend ce risque peut différer d'une culture à l'autre.

En nous basant sur ces observations et sur les résultats obtenus par Beamish (1984), nous pouvons supposer que les deux dimensions du partage d'information, soit le contenu des informations échangées et le processus d'échange, doivent être évalués. On peut supposer que lorsque le partage d'information est jugé important et satisfaisant par les deux partenaires, la performance sera jugée satisfaisante également par ces mêmes partenaires.

\subsection{Partage d'information et performance des coentreprises : y a-t-il un lien direct?}

Les résultats obtenus par Beamish ont été interprétés à la lumière de la théorie des coûts de transaction de Williamson (1981 et 1985). En partageant l'information de façon appropriée, les partenaires d'une coentreprise trouveraient une façon relativement efficiente de diminuer ces coûts de transaction (Hennart, 1988). Cette diminution pourrait donc être considérée comme une variable intermédiaire entre le partage d'information et la performance des coentreprises. Deux problèmes importants se posent lorsqu'on veut évaluer une telle variable.

Le premier problème concerne la définition des coûts de transaction. Les auteurs, qui ont fait appel jusqu'ici à la théorie de Williamson pour expliquer la performance des coentreprises, se sont arrêtés surtout à une des dimensions de l'économie de ces coûts, soit la minimisation des coûts d'acquisition d'information. Or, il semble que deux autres dimensions doivent également être intégrées: la minimisation des coûts de protection des informations confidentielles et la minimisation des coûts de coordination (Contractor et Lorange, 1988). La complexité des coentreprises fait en sorte que ces trois types de coûts de transaction devraient être évalués. L'application de la théorie de Williamson au domaine des coentreprises nécessite selon nous une telle approche globale.

Le second problème a trait à l'opérationnalisation des coûts de transaction. Une bonne part de subjectivité entre dans l'estimation de ces 
coûts. La méthodologie utilisée pour les évaluer doit tenir compte de ce facteur subjectif.

Plutôt que de parler seulement de diminution de coûts, d'autres auteurs ont préféré parler des bénéfices que peuvent retirer les partenaires d'une coentreprise. Par exemple, Shan (1990) cite comme bénéfices ou effets possibles de synergie, l'apprentissage organisationnel, la convergence de technologies et le transfert d'habiletés organisationnelles. Ces effets de synergie, d'ordre plus qualitatif, s'ajouteraient à ceux plus facilement mesurables comme les économies d'échelle et le partage des risques financiers. De tels effets de synergie nécessitent la mise en commun de ressources financières et humaines, mais aussi de connaissances et d'expertise.

Finalement, l'aspect du contrôle sur les décisions à prendre au sein de la coentreprise constitue une variable intermédiaire importante. Les modalités de contrôle décisionnel ou managérial (par exemple, le contrôle partagé par opposition au contrôle dominant) semblent agir sur la réduction des coûts de transaction (Beamish, 1988; Hennart, 1988). Un mode de contrôle partagé favoriserait cette réduction, tandis qu'un mode dominant entraînerait une réduction moindre. Un des facteurs qui favoriserait le partage du contrôle managérial est le fait que les partenaires jugent important et satisfaisant leur façon de partager les informations. En retour, il faut que le flux d'informations ne soit pas bloqué ou diminué par une décision préalable des partenaires d'adopter un mode de contrôle dominant de l'un d'eux.

La relation entre le partage d'information et le mode de contrôle managérial serait donc bidirectionnelle. Notre première hypothèse est donc la suivante:

HYPOTHÈSE la. Un partage de l'information évalué comme important et satisfaisant par les deux partenaires et un contrôle managérial partagé entraînent une forte réduction des coûts de transaction, des effets élevés de synergie et, par conséquent, une bonne performance de la coentreprise.

HYPOTHÈSE $1 b$. Un partage de l'information évalué comme peu important ou non satisfaisant par l'un des partenaires ou par les deux partenaires et un contrôle managérial dominant entraînent une faible réduction des coûts de transaction, peu d'effets de synergie et, par conséquent, une faible performance de la coentreprise. 


\subsection{Le partage de l'information et le contrôle managérial dans les PME}

Julien et Marchesnay (1988) ont insisté sur les caractéristiques qui semblent différencier les PME des grandes entreprises. Par exemple, le contrôle personnel sur la gestion et la flexibilité des PME sont deux caractéristiques qui apparaissent comme complémentaires. L'échange graduel d'informations et l'utilisation de mécanismes de contrôle informels dans les alliances créées par les PME ont été rapportés par Larson (1990) et par Gales et Blackburn (1990). Il serait intéressant de comparer les PME et les grandes entreprises quant à l'utilisation des modes de contrôle managérial au sein des coentreprises. Les quelques données empiriques fournies par Larson et Gales et Blackburn nous amènent à penser que les PME auront moins recours que les grandes entreprises au mode de contrôle formel et dominant sur la coentreprise. Notre deuxième hypothèse est donc la suivante :

HYPOTHÈSE 2. Lorsque l'échange d'information est évalué comme non important ou non satisfaisant, les PME partenaires auront moins recours que les grandes entreprises partenaires à un mode de contrôle formel et dominant sur la coentreprise. Des solutions de remplacement seront alors recherchées.

\section{Méthodologie}

Afin de vérifier nos hypothèses, nous avons opté pour la méthode d'étude de cas. Le faible nombre de coentreprises implantées par les PME québécoises dans les pays en développement explique en partie ce choix : il aurait été difficile de former un échantillon suffisamment grand pour qu'une enquête par questionnaire puisse fournir des résultats valables sur le plan statistique. En outre, la méthode d'étude de cas nous permettait d'étudier le phénomène d'interrelation entre les variables, ainsi que le phénomène d'interaction entre les partenaires. L'évolution dans le temps de ces interactions pouvait alors être mieux comprise.

La méthode d'étude de cas offre, par ailleurs, suffisamment de flexibilité pour nous permettre d'utiliser différents outils de collecte de données: outre l'entrevue, l'observation sur le terrain a aussi été employée. Un des inconvénients de la méthode d'étude de cas est que les analyses quantitatives sont pratiquement impossibles à effectuer. Nous devons donc faire en sorte que les analyses qualitatives des données soient aussi rigoureuses que possible. Pour ce faire, nous avons diversifié nos sources d'information. Nous avons rencontré non seulement les propriétaires-dirigeants, mais aussi, lorsque c'était 
possible, les gestionnaires affectés à la coentreprise ainsi que des tiers qui agissaient à titre de consultants auprès des coentreprises étudiées. Cette diversité de sources d'information peut nous aider à nous protéger contre des biais que certains répondants peuvent avoir, de même que contre nos propres biais. La méthode d'étude de cas en est une interprétative, où habituellement le chercheur interprète directement les données fournies sans s'appuyer d'abord sur des analyses statistiques. La démarche logique est alors plus théorique que statistique pour reprendre l'expression de Yin (1984). En connaissant le degré de convergence entre les différents répondants, cela peut nous aider à nuancer nos analyses.

Il faut évidemment demeurer critique face à la validité externe ou à la généralisation de ces résultats. Notre échantillon est petit et non probabiliste. Même lors des enquêtes effectuées auprès de plus grands échantillons, il est rare que nous puissions satisfaire les conditions strictes de validité externe, en particulier, la condition de sélection au hasard des répondants. Les échantillons non probabilistes sont très fréquents en recherche sur les PME. En conséquence, on peut difficilement généraliser les résultats d'une étude particulière. C'est surtout par les comparaisons entre les résultats d'études différentes (les convergences et les divergences) que nous pourrons progressivement tracer le portrait du phénomène à l'étude.

\subsection{Définition des variables}

\subsubsection{Performance}

La performance des coentreprises a été évaluée subjectivement par chacun des partenaires au cours des entrevues. Nous demandions à chaque partenaire d'évaluer jusqu'à quel point il était satisfait de cette performance («très peu satisfait, moyennement satisfait ou très satisfait»). Étant donné qu'il s'agissait de jeunes coentreprises (âgées de moins de trois ans), le principal critère d'évaluation utilisé par les partenaires n'était pas le montant des bénéfices réalisés (qui est un des critères financiers fréquemment utilisés dans la littérature), mais plutôt la minimisation des pertes financières et l'atteinte graduelle du seuil de rentabilité. Dans tous les cas étudiés, la coentreprise était considérée comme un investissement à long terme et des pertes financières étaient prévues pendant les premières années d'opération.

\subsubsection{Partage d'information}

Cette variable était étudiée sous deux aspects : le contenu des informations échangées et le processus d'échange d'information. 
En ce qui concerne le contenu des informations échangées, nous demandions à chaque répondant de nous dire jusqu'à quel point il considérait important d'acquérir ou de fournir des informations à son partenaire dans chacun des domaines suivants : connaissance de l'environnement, recherche et développement, production et opérations, gestion des ressources humaines, marketing, finance, outils de gestion en général.

Le processus d'échange d'information était évalué par le biais de questions visant à permettre graduellement à chaque répondant d'exprimer ses satisfactions et insatisfactions au regard de la manière dont l'autre partenaire demandait ou fournissait des informations. Les trois aspects relevés plus haut dans la recension de la documentation ont été abordés : le synchronisme des échanges, le degré d'ouverture et d'adaptation des partenaires et le degré d'opportunisme.

\subsubsection{Formes de contrôle managérial}

Pour chacune des fonctions de la coentreprise, nous demandions aux partenaires si les décisions étaient habituellement conjointes ou prises par l'un d'eux. Nous tentions ensuite d'arriver à un diagnostic d'ensemble quant à la forme de contrôle managérial qui caractérisait le mieux chaque coentreprise.

\subsubsection{Coûts de transaction}

Pour chacun des domaines où le partenaire déclarait important l'échange d'information, nous tentions de déterminer avec lui les divers coûts en temps, en argent et en efforts engendrés par:

- l'acquisition d'information auprès du partenaire;

- la protection des informations confidentielles;

- la coordination avec son partenaire.

Les coûts de transaction, tout comme les effets de synergie, étaient évalués de façon indirecte. À partir des propos exprimés en entrevue par les répondants quant aux difficultés et aux avantages de leur expérience de partenariat, nous faisions des inférences aussi justes que possible quant à l'importance des coûts de transaction et des effets de synergie.

\subsubsection{Effets de synergie}

Pour chacun des domaines où le partenaire déclarait important l'échange d'information, nous tentions de déterminer avec lui quels étaient les divers bénéfices ou effets de synergie. Ceux-ci pouvaient être exprimés en termes 
quantitatifs (p. ex., économies d'échelle) ou qualitatifs (p. ex., adaptation plus rapide à l'environnement).

\subsection{Choix des répondants}

Sept coentreprises forment notre échantillon. Les partenaires québécois de ces coentreprises ont été contactés par le biais de revues spécialisées ou sur recommandation de consultants. Le tableau 1 présente les principales caractéristiques des partenaires et des coentreprises étudiées. Parmi les sept coentreprises, une seule, soit le cas $\mathrm{n}^{\circ} 2$ constitue une grande entreprise dans son secteur d'activités (éducation - collèges privés). Les données obtenues de la part du partenaire québécois de cette coentreprise ont été comparées aux données obtenues dans la coentreprise $\mathrm{n}^{\circ} 1$ afin de vérifier la deuxième hypothèse de notre étude (permettant la comparaison PME - grandes entreprises). Ces deux cas étaient des collèges privés.

Le cas $\mathrm{n}^{\circ} 4$ est un cas particulier dans notre étude. Le répondant québécois que nous avons rencontré pour l'entrevue est le président-directeur général d'un consortium québécois qui regroupe six entreprises, dont des grandes entreprises. Ce répondant est l'initiateur et le maître d'œuvre de la formation du consortium québécois qui a créé une coentreprise au Maroc; il est celui qui est le plus impliqué dans la gestion de la coentreprise. Les autres partenaires sont surtout des investisseurs et des firmes qui fournissent une expertise technologique. Étant donné que ce répondant dirige une PME, nous avons supposé que le consortium en question pouvait se comporter au Maroc autant comme une PME que comme une grande entreprise. L'analyse de cas nous renseignera à ce sujet-là.

Six coentreprises sur sept œuvraient dans le secteur des services (écoles, vente et installation d'équipement au gaz, services agro-alimentaires, services de gestion et édition). La seule coentreprise manufacturière de notre échantillon œuvrait dans la fabrication de machinerie industrielle. L'hétérogénéité des entreprises choisies s'explique par le fait qu'au Québec, le nombre de PME qui implantent des coentreprises dans les pays en développement est encore restreint et qu'il était donc impossible de choisir un nombre suffisant d'entreprises dans un secteur en particulier.

\subsection{Formes de rencontres et d'analyse des résultats}

Les entrevues en profondeur avec chacun des responsables québécois des coentreprises ont été faites entre novembre 1992 et février 1993. Par la suite, nous avons demandé aux partenaires établis au Maroc et en Tunisie s'ils acceptaient que nous contactions leurs vis-à-vis dans ces pays en vue d'une 
TABleau 1

Caractéristiques des partenaires et des entreprises conjointes étudiées

\begin{tabular}{|c|c|c|c|c|c|c|c|c|c|c|c|c|}
\hline Cas & $\mathbf{N}$ & $\begin{array}{l}\text { Partenaires québécois } \\
\text { Secteur d'activités }\end{array}$ & Empl. & $\bar{N}$ & Pays & $\begin{array}{l}\text { Partenaires étrangers } \\
\text { Secteur d'activités }\end{array}$ & Empl. & Pays & Âge & $\begin{array}{l}\text { Entreprises conjointes } \\
\text { Secteur d'activités }\end{array}$ & Empl. & $\begin{array}{l}\text { Répartition des } \\
\text { actions }\end{array}$ \\
\hline 1 & 1 & - Services: éducation (collège privé) & 90 & 4 & Maroc & $\begin{array}{l}\text { - (1) Services (immobiliers)** } \\
\text { - (3) Individus (investisseurs } \\
\text { passifs) }\end{array}$ & 12 & Maroc & 1,5 an & $\begin{array}{l}\text { Services (éducation); collège } \\
\text { privé }\end{array}$ & 20 & $\begin{array}{l}50 \% \text { québécois } \\
35 \% \text { partenaire } \\
\text { principal marocain } \\
5 \% \text { chacun } \\
3 \text { investisseurs } \\
\text { passifs }\end{array}$ \\
\hline 2 & 1 & - Services; éducation (collège privé) & 250 & 2 & Maroc & $\begin{array}{l}\text { - (1) Services (education)** } \\
\text { - (1) Individu (investisseur } \\
\text { passif) }\end{array}$ & 15 & Maroc & 1,5 an & $\begin{array}{l}\text { Services (éducation); collège } \\
\text { privé }\end{array}$ & 15 & $\begin{array}{l}50 \% \text { québécois } \\
40 \% \text { partenaire } \\
\text { principal marocain } \\
10 \% \\
\text { investissement } \\
\text { passif }\end{array}$ \\
\hline 3 & 1 & $\begin{array}{l}\text { - Manufacturier: fabrication } \\
\text { d'équipement de levage et } \\
\text { manutention }\end{array}$ & 45 & $1^{*}$ & Tunisie & $\begin{array}{l}\text { - Manufacurier: fabrication de } \\
\text { structures métalliques }\end{array}$ & 80 & Tunisie & 3 ans & $\begin{array}{l}\text { Équipement de levage et } \\
\text { manutention }\end{array}$ & 20 & $\begin{array}{l}40 \% \text { québécois } \\
60 \% \text { tunisiens }\end{array}$ \\
\hline 4 & 6 & $\begin{array}{l}\text { - Services (gestion) } \\
\text { - Vente et installation d'équipement au } \\
\text { gaz; distribution de produits } \\
\text { pétroliers } \\
\text { - Services (financement) } \\
\text { - Services (ingénierie) } \\
\text { - Manufacturier (fabrication } \\
\text { d'équipement au gaz) } \\
\text { - Construction }\end{array}$ & $\begin{array}{r}50^{* *} \\
3000 \\
250 \\
200 \\
200 \\
150\end{array}$ & $1^{*}$ & Maroc & $\begin{array}{l}\text { - Distribution de produits } \\
\text { pétroliers }\end{array}$ & 70 & Maroc & 1,5 an & $\begin{array}{l}\text { Services (installation } \\
\text { d'équipement et distribution de } \\
\text { produits pétroliers) }\end{array}$ & $e^{15}$ & $\begin{array}{l}25 \% \text { québécois } \\
75 \% \text { marocains }\end{array}$ \\
\hline 5 & 1 & $\begin{array}{l}\text { - Services de consultation dans le } \\
\text { domaine agro-alimentaire }\end{array}$ & 8 & $1^{*}$ & Maroc & - Production agricole & 55 & Maroc & 1 an & Producion agricole & 12 & $\begin{array}{l}90 \% \text { québécois } \\
10 \% \text { marocains }\end{array}$ \\
\hline 6 & 1 & - Services (gestion) & 35 & 1 & Cameroun & - Services (gestion) & 6 & Cameroun & $\begin{array}{l}10 \\
\text { mois }\end{array}$ & Services (gestion) & 6 & $\begin{array}{l}60 \% \text { québécois } \\
40 \% \text { marocains }\end{array}$ \\
\hline 7 & 1 & - Édition & 30 & 4 & $\begin{array}{l}\text { Côte- } \\
\text { d'Ivoire }\end{array}$ & $\begin{array}{l}\text { - Services (agence } \\
\text { gouvernementale } \\
\text { - Individus (investisseurs } \\
\text { passifs) }\end{array}$ & & $\begin{array}{l}\text { Côte- } \\
\text { d'Ivoire }\end{array}$ & 1,5 an & Édition & 26 & $\begin{array}{l}40 \% \text { français } \\
10 \% \text { québécois }\end{array}$ \\
\hline & & & & 1 & France & - Édition & 400 & & & & & \\
\hline
\end{tabular}


rencontre sur place. Deux partenaires marocains et un partenaire tunisien ont pu ainsi être rencontrés. Le Maroc et la Tunisie ont été choisis parce que c'est là qu'était concentré le plus grand nombre de partenaires étrangers. Dans chacune des coentreprises visitées, nous avons pu rencontrer le gestionnaire québécois affecté aux opérations. Ces gestionnaires nous ont fourni des renseignements très précieux sur la réalité vécue dans les coentreprises. Ces renseignements complétaient et, dans certains cas, nuançaient les évaluations fournies par les propriétaires-dirigeants.

Les entrevues ont duré entre 1 heure et demie et 2 heures, selon la disponibilité des répondants. Nous nous assurions que tous les domaines inscrits dans notre grille d'évaluation avaient été abordés. Cette grille était utilisée non seulement pour s'assurer que toutes les questions spécifiques à notre étude soient posées, mais aussi pour inscrire au fur et à mesure les réponses fournies.

Étant donné que l'évaluation des variables «coûts de transaction » et «effets de synergie » nécessitait une bonne part d'interprétation, nous avons tenté de baser nos interprétations sur des événements concrets rapportés par les répondants. Ceux-ci étaient donc assez libres de raconter leur vécu avec la coentreprise. Les répondants québécois se sont montrés spontanés dans leurs façons d'aborder les difficultés et les avantages de la coentreprise. Quant aux partenaires étrangers, à l'exception du cas $\mathrm{n}^{\circ} 1$, les renseignements fournis étaient habituellement imprécis au début; ces répondants voulaient d'abord échanger sur des sujets qui n'avaient pas un rapport direct avec la coentreprise. Par la suite, les renseignements se sont faits un peu plus précis.

Voici quelques exemples d'événements concrets sur lesquels nous nous sommes basés pour évaluer les coûts de transaction et les effets de synergie. Dans le cas du collège privé maroco-canadien qui constitue le cas $n^{\circ} 1$ de notre étude, le partenaire québécois jugeait important l'acquisition d'information sur la clientèle potentielle, sur les aspects de logistique et de relations avec l'administration marocaine, ainsi que sur le financement local de l'entreprise conjointe. La contribution du partenaire québécois consistait en son expertise en matière de pédagogie et de gestion des ressources humaines. Or, des problèmes de communication entre les partenaires ont fait en sorte que la clientèle potentielle a été surestimée, que le partenaire québécois a dû s'occuper de questions logistiques à la place du partenaire marocain et que des possibilités de financement local auraient été perdues lors de la première année d'opération. Ce sont là des événements rapportés par le partenaire québécois. De son côté, le partenaire marocain rapporte que les méthodes pédagogiques mises de l'avant par le partenaire québécois sont mal expliquées et provoquent du ressentiment chez les étudiants qui ont recours à différents moyens de pression (p. ex., refus de se présenter aux examens, menaces de manifestations). Devant le refus du partenaire québécois de modifier son approche pédagogique, le 
partenaire marocain a décidé de lui fournir encore moins d'information. Ces événements et d'autres propos rapportés par les partenaires et les gestionnaires nous ont amenés à conclure que les coûts d'acquisition d'information et de coordination demeuraient élevés pour les deux partenaires, tandis que les effets de synergie sont nuls.

Après chacune des entrevues avec les répondants, les données étaient interprétées à la lumière des hypothèses de recherche. Un rapport préliminaire d'entrevue était établi dans les jours suivant l'entrevue. Une copie de ce rapport était envoyée à chaque répondant afin d'en vérifier les principales conclusions. Nous contactions à nouveau par téléphone chaque partenaire afin qu'il puisse commenter le rapport et nous aider à le compléter et à le rendre plus conforme à la réalité.

L'objectif visé lors de ces échanges avec les répondants était de pouvoir coter de façon sommaire les réponses fournies. La grille d'évaluation contient les cotations des réponses pour chacune des variables. Ces cotations sont moins exhaustives que dans les enquêtes par questionnaire. Elles ne servaient qu'à établir un ordre de grandeur, et non pas à calculer des valeurs statistiques. Par ailleurs, la nature de certaines de nos variables, par exemple, celle des coûts de transaction, permet difficilement une enquête par questionnaire. C'est par le biais de questions progressives en cours d'entrevue que nous avons tenté, avec l'aide de chaque répondant, d'établir des cotes pour chaque variable.

\section{Résultats}

Nous présenterons tout d'abord un aperçu d'ensemble des résultats. Le tableau 2 nous aidera à mieux les saisir. Nous présenterons également une analyse des relations entre les variables à la lumière des hypothèses de recherche. Le tableau 3 résume cette analyse des interrelations.

\subsection{Aperçu d'ensemble des résultats}

En ce qui concerne le contenu des informations échangées, les partenaires québécois estiment importants les échanges d'information dans les domaines suivants :

- la finance-comptabilité, en particulier le contrôle interne: le manque de rigueur des méthodes de contrôle comptable est un problème fréquemment soulevé ;

- la gestion des opérations, notamment le contrôle de la qualité des biens et services, souvent perçue comme problématique, ainsi que l'amélioration de la productivité des travailleurs locaux ; 
- la gestion des ressources humaines : la question de la formation technique déficiente et des attitudes des travailleurs locaux (p. ex., manque d'attention ou de motivation) revient souvent dans les échanges entre partenaires ;

- le marketing: c'est le domaine où les apports des partenaires étrangers sont les plus importants;

- l'environnement củlturel, politique et administratif du pays hôte.

Quant aux trois partenaires étrangers rencontrés, ils recherchent surtout des informations dans les domaines suivants:

- production et distribution (technologie);

- la gestion des ressources humaines (amélioration de la productivité ou encore du climat de travail).

En ce qui concerne le processus d'échange d'information, la majorité des partenaires québécois se disent satisfaits ou assez satisfaits de la façon dont se déroulent ces échanges, à l'exception du cas $\mathrm{n}^{\circ} 1$ où le manque d'ouverture du partenaire marocain est rapporté. Celui-ci en retour se plaint du manque d'adaptation culturelle du partenaire québécois, ainsi que du gestionnaire québécois affecté aux opérations sur place.

\subsubsection{Formes de contrôle managérial}

Cinq des sept coentreprises avaient adopté un mode de contrôle managérial partagé; ce sont les cinq coentreprises dont la performance était évaluée comme étant satisfaisante. Les cas 1 et 2 sont ceux où un contrôle managérial dominant a été utilisé et où la performance était jugée si insatisfaisante par le partenaire québécois que la coentreprise était au moment de l'étude soit dissoute (cas $\mathrm{n}^{\circ} 2$ ), soit en instance de dissolution ( $\operatorname{cas} \mathrm{n}^{\circ} 1$ ).

\subsubsection{Réductions des coûts de transaction}

Les données recueillies indiquent que les coûts de transaction reliés à l'acquisition d'information externe sont ceux qui doivent être diminués rapidement lors de l'implantation de la coentreprise si l'on veut que celle-ci survive. Ce sont des coûts surtout évalués en termes de temps plutôt qu'en termes d'efforts d'adaptation socioculturelle. Les informations échangées sont alors principalement factuelles (p. ex., où trouver tel type de fournisseurs).

Après les premiers mois d'implantation de la coentreprise, ce sont les coûts de coordination qui deviennent plus importants. Chaque partenaire doit alors faire des efforts d'introspection et d'adaptation afin d'abord d'informer 
TABLEAU 2

Aperçu d'ensemble des résultats

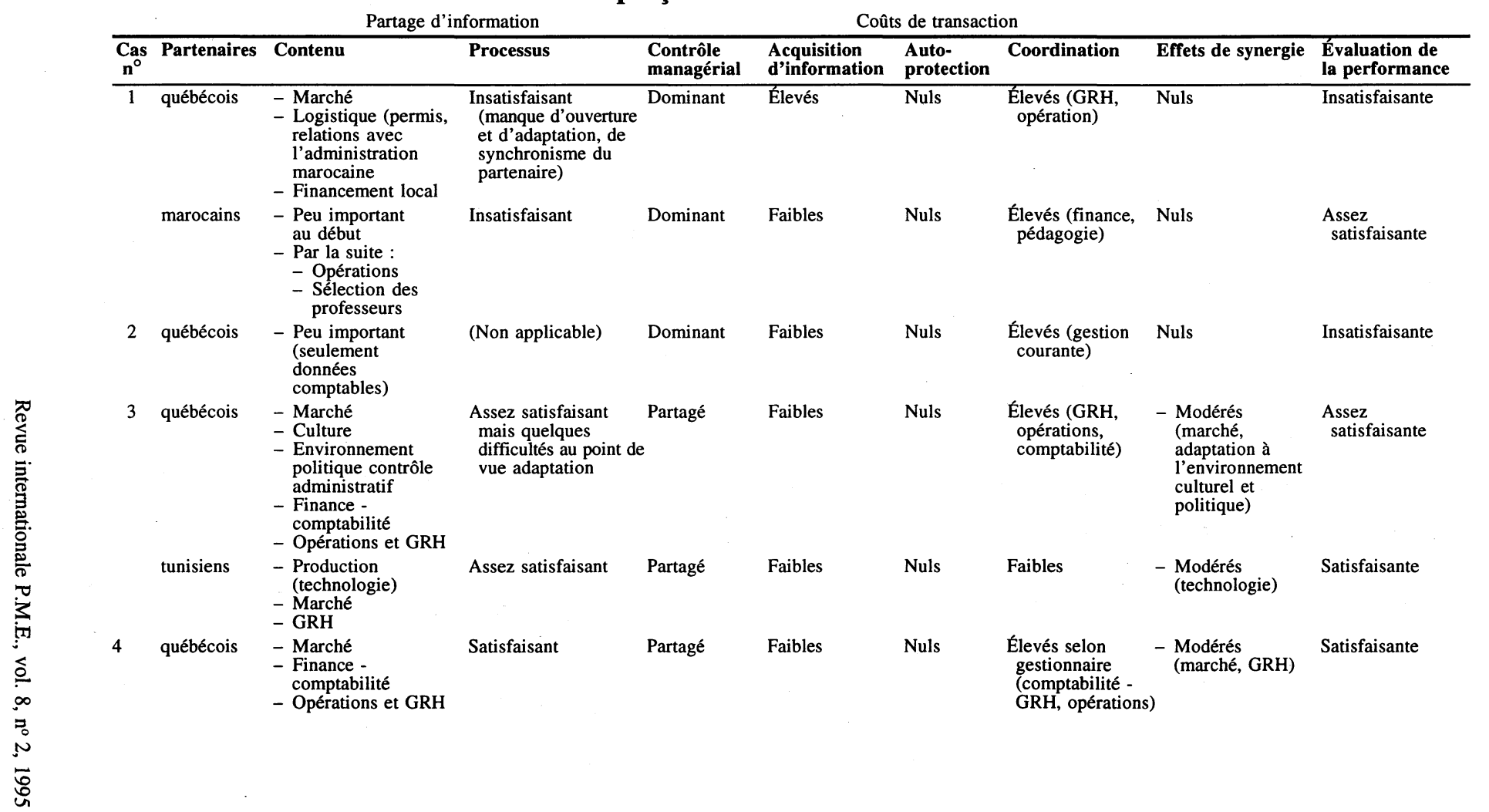


TABleau 2 (suite)

Aperçu d'ensemble des résultats

Partage d'information

Coûts de transaction

\begin{tabular}{|c|c|c|c|c|c|c|c|c|c|}
\hline $\begin{array}{c}\text { Cas } \\
n^{\circ}\end{array}$ & Partenaires & Contenu & Processus & $\begin{array}{l}\text { Contrôle } \\
\text { managérial }\end{array}$ & $\begin{array}{l}\text { Acquisition } \\
\text { d'information }\end{array}$ & $\begin{array}{l}\text { Auto- } \\
\text { protection }\end{array}$ & Coordination & Effets de synergie & $\begin{array}{l}\text { Évaluation de } \\
\text { la performance }\end{array}$ \\
\hline & marocains & $\begin{array}{l}\text { - Marché } \\
\text { - Production } \\
\text { (technologie) } \\
\text { - GRH } \\
\text { - Outils de gestion } \\
\text { restructuration } \\
\text { du groupe }\end{array}$ & Satisfaisant & Partagé & Faibles & Nuls & Faibles & $\begin{array}{l}\text { - Élevés (GRH, } \\
\text { opérations, } \\
\text { apprentissages } \\
\text { généralisables) }\end{array}$ & Satisfaisante \\
\hline 5 & québécois & $\begin{array}{l}\text { - Marché } \\
\text { - GRH } \\
\text { - Comptabilité } \\
\text { - Environnement } \\
\text { politique }\end{array}$ & Satisfaisant & Partagé & Faibles & Nuls & Faibles & Modérés (marché) & Satisfaisante \\
\hline 6 & québécois & $\begin{array}{l}\text { - Culture } \\
\text { - Marché } \\
\text { - Savoir-faire en } \\
\text { management }\end{array}$ & Satisfaisant & Partagé & Faibles & Nuls & Faibles & $\begin{array}{l}\text { Élevés (marché, } \\
\text { constitution d'un } \\
\text { réseau, présence } \\
\text { en Afrique sans } \\
\text { investissements } \\
\text { trop importants) }\end{array}$ & Satisfaisante \\
\hline 7 & québécois & $\begin{array}{l}\text { - Comptabilité } \\
\text { - Marché } \\
\text { - Adaptation du } \\
\text { produit }\end{array}$ & Satisfaisant & Partagé & Faibles & Nuls & Faibles & $\begin{array}{l}\text { Élevés (avec le } \\
\text { partenaire } \\
\text { français); } \\
\text { adaptation du } \\
\text { produit et marché }\end{array}$ & Satisfaisante \\
\hline
\end{tabular}


TABleau 3

Évaluation des résultats par rapport aux hypothèses de recherche Échec de la coentreprise Performance jugée satisfaisante

\begin{tabular}{|c|c|c|c|}
\hline & \multicolumn{3}{|c|}{ Hypothèses } \\
\hline & $1-\mathbf{a}$ & $1-b$ & 2 \\
\hline Cas no 1 & & $\begin{array}{l}\text { - confirmée } \\
\text { (partenaire } \\
\text { québécois) } \\
\text { - non confirmée } \\
\text { (partenaire } \\
\text { étranger) }\end{array}$ & $\begin{array}{r}\text { - confirmée } \\
\text { (partenaire } \\
\text { québécois) }\end{array}$ \\
\hline Cas $n^{0} 2$ & & $\begin{array}{l}\text { - partiellement } \\
\text { confirmée } \\
\text { (partenaire } \\
\text { québécois) }\end{array}$ & $\begin{array}{r}\text { - confirmée } \\
\text { (partenaire } \\
\text { québécois) }\end{array}$ \\
\hline Cas $n^{0} 3$ & $\begin{array}{l}\text { - partiellement } \\
\text { confirmée } \\
\text { (partenaire } \\
\text { québécois) } \\
\text { - confirmée } \\
\text { (partenaire } \\
\text { étranger) }\end{array}$ & & \\
\hline Cas no 4 & $\begin{array}{l}\text { - partiellement } \\
\text { confirmée } \\
\text { (partenaire } \\
\text { québécois) } \\
\text { - confirmée } \\
\text { (partenaire } \\
\text { étranger) }\end{array}$ & & \\
\hline Cas no 5 & $\begin{array}{r}\text { - confirmée } \\
\text { (partenaire } \\
\text { québécois) }\end{array}$ & & \\
\hline $\operatorname{Cas} n^{\circ} 6$ & $\begin{array}{r}\text { - confirmée } \\
\text { (partenaire } \\
\text { québécois) }\end{array}$ & & \\
\hline $\operatorname{Cas} n^{\circ} 7$ & $\begin{array}{c}\text { - confirmée } \\
\text { partenaire } \\
\text { québécois) }\end{array}$ & & \\
\hline $\begin{array}{l}\text { Évaluation } \\
\text { d'ensemble }\end{array}$ & $\begin{array}{l}\text { - partiellement } \\
\text { confirmée } \\
\text { (dissymétrie entre } \\
\text { les partenaires } \\
\text { québécois et } \\
\text { étrangers) }\end{array}$ & $\begin{array}{l}\text { - partiellement } \\
\text { confirmée } \\
\text { (dissymétrie entre } \\
\text { les partenaires } \\
\text { québécois et } \\
\text { étrangers) }\end{array}$ & - confirmée \\
\hline
\end{tabular}


son vis-à-vis quant à ses méthodes de gestion, puis comprendre les méthodes de l'autre et s'y ajuster. Les informations échangées et utilisées sont alors moins factuelles et exigent plus d'efforts d'adaptation socioculturelle de chaque partenaire. Au fur et à mesure que la coentreprise évolue, chaque partenaire interprète ce qui s'y passe de façon parfois complètement différente de son vis-à-vis. S'ils ne confrontent pas leurs interprétations, au risque de créer des mini-crises comme nous le constaterons plus loin, les partenaires s'exposent à prendre des positions de plus en plus divergentes face à leur «bébé ». C'est ce qui est arrivé aux cas 1 et 2 . Pour les cas 3 et 4 , des crises sérieuses ont été vécues; grâce en partie à la souplesse des gestionnaires québécois affectés à ces deux dernières coentreprises, ces crises ont pu être résorbées, non sans changements majeurs dans l'organisation de la coentreprise.

Parmi les domaines où les coûts de coordination sont souvent élevés, ceux de la gestion des opérations et de la gestion des ressources humaines sont particulièrement importants, surtout aux yeux des gestionnaires québécois interrogés. Les conceptions souvent différentes de la qualité et de la productivité entre les Québécois et les Marocains ou les Tunisiens obligent les gestionnaires québécois à trouver des façons de faire accepter par les employés des modifications importantes dans leurs façons de travailler.

En ce qui concerne les coûts de protection des informations confidentielles, ils sont évalués comme étant nuls par les partenaires québécois et étrangers interrogés. Il faut dire cependant qu'il est délicat pour les répondants d'aborder ce sujet. Nous pouvons difficilement conclure que ces coûts soient négligeables sur la seule base des données de notre étude.

\subsubsection{Effets de synergie}

Les partenaires des coentreprises 1 et 2 ne perçoivent pas d'effets de synergie liés au partenariat. Dans les autres cas, c'est surtout dans le domaine de la mise en marché et de la gestion des ressources humaines que le partenariat semble profitable aux partenaires. Le fait que les coentreprises étudiées soient relativement jeunes (moins de trois ans) peut expliquer le peu d'effets de synergie rapportés par les partenaires dans d'autres domaines d'activité.

\subsection{Interrelations entre les variables}

Dans les deux coentreprises non performantes, les interrelations entre les variables évaluées auprès des répondants québécois confirment partiellement $l^{\prime}$ hypothèse $1 b$. Le répondant québécois de la coentreprise $n^{\circ} 2$ évaluait les coûts d'acquisition d'information comme étant faibles, alors que selon notre 
hypothèse $1 b$, ces coûts auraient dû être élevés. L'évaluation des autres variables va dans le sens prédit par l'hypothèse $1 b$.

Dans la coentreprise $n^{\circ} 1$, les données recueillies auprès du partenaire marocain ne confirment pas l'hypothèse $1 \mathrm{~b}$. Il y a dissymétrie entre les partenaires quant à leur évaluation de la performance : celle-ci est évaluée comme étant assez satisfaisante par le partenaire marocain, alors que le partenaire québécois la juge si insatisfaisante qu'il a déjà amorcé les procédures visant à y mettre fin. Cela montre à quel point l'évaluation de la performance d'une même coentreprise peut varier selon les critères adoptés par les partenaires. Même si les deux partenaires considéraient la coentreprise comme un investissement à long terme, ils différaient quant à la conception de ce qui constituait une perte financière raisonnable dans ce contexte de coentreprise nouvellement établie. Pour les deux partenaires, il s'agissait d'un échec compte tenu de la mésentente qui s'est installée progressivement dans la coentreprise. Le même constat d'échec a été fait par le partenaire québécois de la coentreprise $n^{\circ} 2$.

Dans les cinq coentreprises performantes, les interrelations entre les variables évaluées auprès des répondants québécois confirment partiellement l'hypothèse 1a. Les coûts de coordination sont évalués comme étant élevés par les répondants des coentreprises 3 et 4 , alors qu'ils auraient dû être bas selon notre hypothèse 1a. L'évaluation des autres variables va dans le sens prédit par notre hypothèse 1a. Chez les répondants étrangers des deux coentreprises performantes que nous avons pu visiter, les interrelations entre les variables évaluées confirmaient entièrement l'hypothèse $1 \mathrm{a}$.

La présence de coûts élevés de coordination, même dans des coentreprises évaluées comme satisfaisantes sur le plan de la performance, alliée au fait que les coûts d'acquisition d'information sont faibles dans ces mêmes coentreprises, démontre jusqu'à quel point il est important de distinguer entre les différents types de coûts de transaction. Sans une telle distinction, les tentatives d'explication des coentreprises par la théorie de Williamson risquent d'être incohérentes d'une étude à l'autre. La réduction des coûts de transaction, selon nos données, doit être considérée en fonction du temps. Comme nous le mentionnions plus haut, la réduction des coûts d'acquisition d'information doit survenir assez tôt selon les répondants rencontrés; ceux-ci s'attendent à une réduction assez rapide grâce à l'apport du partenaire. Par la suite, c'est la réduction des coûts de coordination qui devient plus importante. La figure 1 présente notre analyse théorique de la réduction des coûts de transaction et de l'augmentation des bénéfices en fonction du temps, facteur qui n'avait pas été intégré dans nos hypothèses. 
La période qui précède la signature du contrat de coentreprise est propice à l'acquisition des premières informations sur le partenaire et sur l'environnement du pays visé. Pour certains partenaires québécois rencontrés, une bonne partie des informations sur l'environnement du pays visé avaient déjà été acquises pendant cette phase. Il s'agit des partenaires des coentreprises 4 et 7.

La deuxième phase débute avec la signature de l'entente d'entreprise conjointe. Dans les coentreprises étudiées, à l'exception de la coentreprise 2 et 6 , il y a eu une période de rodage. Les opérations de fabrication ou de distribution ne peuvent débuter tout de suite parce que l'entreprise n'a pas encore reçu tout l'équipement nécessaire, ou parce que les employés n'ont pas encore tous été embauchés. L'acquisition d'information se poursuit alors à un rythme plus accéléré. Déjà, à cette deuxième phase, des problèmes étaient vécus dans la coentreprise $n^{\circ} 1$. À cause d'informations déficientes de la part du partenaire principal marocain, le directeur québécois a dû à l'occasion se rendre lui-même au Maroc pour faire dédouaner de l'équipement nécessaire à l'implantation de l'entreprise maroco-canadienne.

Après l'obtention des premiers contrats, des premières commandes ou encore les premières inscriptions d'élèves débutent vraiment les opérations. Cette troisième phase est celle des premiers tests sérieux de la capacité de coordination des parties impliquées. Selon le rythme de réduction des coûts de coordination, les partenaires évalueront le fonctionnement et la performance de la coentreprise comme étant satisfaisants ou non. Ils pourront, dans une quatrième phase, consolider la coentreprise, la réorganiser, la réorienter ou y mettre fin. Par exemple, dans le cas de la coentreprise $n^{\circ} 1$, il s'agit d'une réorientation selon l'expression même des partenaires; un de ces derniers va racheter les actions de l'autre pour, probablement, les revendre à un autre partenaire. Dans le cas des coentreprises 3 et 4 , une réorganisation a été nécessaire à ce stade; par exemple, le directeur de la firme partenaire a été écarté du pouvoir dans le premier cas et limogé dans le second.

L'augmentation des bénéfices ou effets de synergie semble se faire lentement jusqu'à ce que la coordination entre les parties devienne plus facile.

Il peut paraître surprenant de constater qu'aucun des partenaires rencontrés n'a déclaré que les coûts d'autoprotection étaient importants dans leur cas. Comme nous le mentionnions plus haut, c'est probablement un sujet trop délicat pour être abordé dans une étude comme celle-ci. Peut-être qu'une recherche-action ou recherche participative qui donnerait la chance au chercheur d'observer de l'intérieur même des entreprises serait plus efficace pour évaluer cette variable. 


\section{FIGURE 1}

\section{Analyse théorique de la réduction des coûts de transaction} et de l'augmentation des bénéfices en fonction du temps

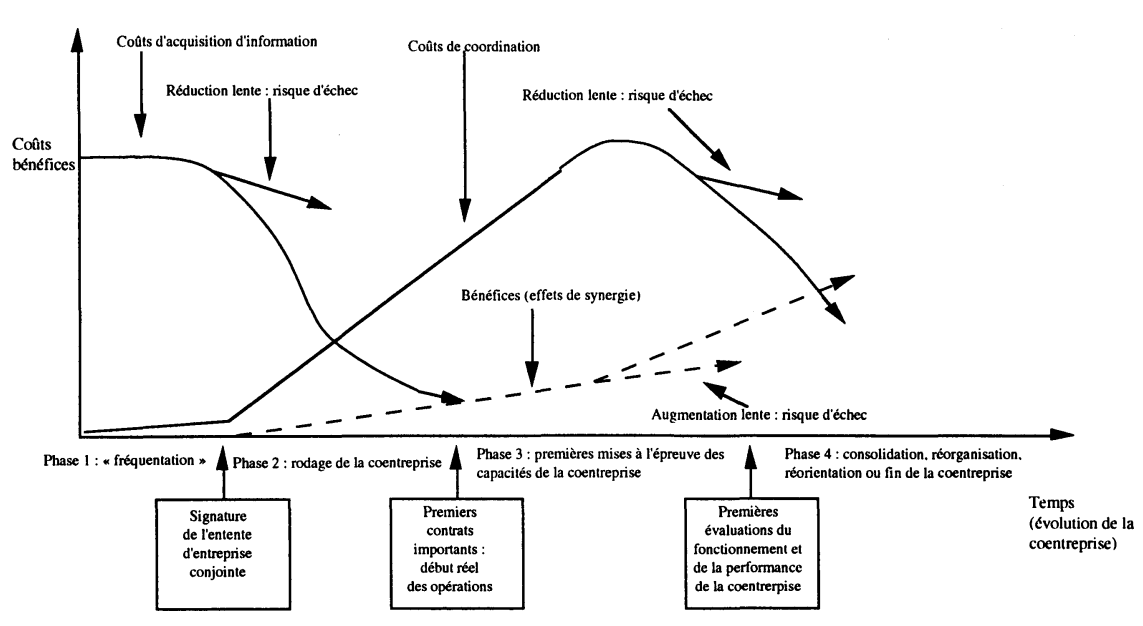

Dans les deux coentreprises non performantes, l'hypothèse 2 est confirmée. La PME partenaire préférait opter pour un nouveau partenariat plutôt que pour la création d'une filiale détenue à $100 \%$, tandis que la grande entreprise partenaire préférait cette dernière possibilité.

Évidemment, il ne s'agit là que de deux cas. D'autres études empiriques qui incluraient un plus grand nombre de PME conjointes et de GE conjointes pourraient nous renseigner davantage sur les comportements des PME et des grandes entreprises face à une performance insatisfaisante de leur coentreprise. Si ces études empiriques arrivaient aux mêmes résultats que les nôtres, il faudrait se demander pourquoi les PME optent pour un autre partenariat plutôt que pour une filiale détenue à $100 \%$. Est-ce à cause d'un manque de ressources (financières, humaines, techniques)? Ce n'est peut-être pas seulement une question de ressources. Par exemple, le directeur de la PME qui fait partie du puissant consortium québécois agissant comme partenaire dans la coentreprise $n^{\circ} 4$ nous a dit qu'en cas de performance insatisfaisante, la première solution envisagée serait la recherche d'un autre partenaire marocain.

Il est possible que certaines caractéristiques propres aux PME expliquent également leur préférence pour un nouveau partenariat. Nous mentionnions plus haut que le contrôle personnalisé de l'entreprise par son propriétairedirigeant ainsi que la flexibilité sont deux caractéristiques que l'on peut retrouver dans un grand nombre de PME. Ces caractéristiques semblent 
s'appliquer jusqu'à un certain point aux alliances entre PME, où les rapports personnels entre les responsables semblent plus déterminants que l'application formelle de contrats (Larson, 1990).

Devant un constat de performance insatisfaisante de la coentreprise dans un pays en développement, une PME québécoise qui déciderait d'assumer seule le contrôle managérial de cette entreprise aurait probablement plus de difficultés à maintenir un mode de contrôle plus personnalisé, plus simple et plus informel que celui observé dans les grandes entreprises en général. Ne pouvant exercer lui-même une supervision directe à cause de l'éloignement de la coentreprise et de l'information souvent tardive et incomplète qu'il reçoit, et ne pouvant, par ailleurs, pas compter sur un partenaire pour assumer une partie de cette supervision directe, le propriétaire-dirigeant de PME se verrait forcé de recourir à des formes de contrôle avec lesquelles il est moins familier.

Les propriétaires-dirigeants espèrent alors trouver un nouveau partenaire qui partagera leur vision de la coentreprise et avec lequel ils tenteront d'en continuer la gestion de façon aussi directe et personnelle que possible. Les compromis que le partenariat les oblige à faire - par exemple, partage du pouvoir décisionnel, augmentation des coûts de coordination - sont compensés d'abord par une économie en investissements et aussi une économie dans l'acquisition d'informations externes. Ces compromis sont ensuite compensés, lorsque le «mariage» s'avère un succès, par le maintien des capacités de flexibilité et d'interactivité pour faire face conjointement à la complexité et à la possible turbulence environnementale (rapidité de réaction).

\section{Conclusion}

Les résultats que nous avons recueillis suggèrent que le lien entre le partage d'information et la performance des coentreprises sera mieux compris si l'on tient compte des variables intermédiaires identifiées dans cette étude : les coûts de transaction, les effets de synergie et la forme de contrôle managérial utilisée dans la coentreprise. Ce lien semble varier en fonction du temps. Ce facteur de temps n'a pas été pris en considération par les auteurs comme Beamish et Hennart qui ont avant nous tenté d'établir un lien entre le partage d'information et la performance des coentreprises. Selon le stade d'évolution de la coentreprise, certains types de coûts de transaction doivent être diminués plus rapidement que d'autres. Il aurait été intéressant de pouvoir poursuivre l'étude avec les mêmes coentreprises et de vérifier si les effets de synergie obtenus et la forme de contrôle managérial utilisée dans les coentreprises évoluent également en fonction du temps. 
Il nous faut être prudents dans l'interprétation des résultats de cette étude, en partie à cause de la variété des secteurs d'activité de nos coentreprises. Ainsi, les deux coentreprises non performantes œuvraient dans le secteur de l'éducation (collèges privés), lequel semble présenter au Maroc des caractéristiques assez différentes des secteurs d'activité des autres coentreprises, tant par la forte concurrence qui semble prévaloir, que par la difficulté d'obtenir les permis et de satisfaire aux exigences des bureaucrates. En plus des différences de secteur, des différences en termes d'objectifs stratégiques existaient entre ces deux coentreprises non performantes et les autres plus performantes. En effet, alors que la plupart des coentreprises performantes étaient destinées à devenir progressivement plus autonomes, ou encore à être prises progressivement en charge par le partenaire étranger, les deux coentreprises non performantes étaient au contraire organisées de façon à maintenir une présence d'enseignants québécois, et dans le cas de la coentreprise $n^{\circ} 1$, d'un codirecteur québécois. Cela, d'ailleurs, était une des sources principales de conflit entre le partenaire marocain et le gestionnaire québécois dans le cas de la coentreprise $n^{\circ} 1$.

Étant donné que notre étude portait sur les processus de gestion dans la coentreprise, nous ne pouvions étudier de façon systématique les variables reliées aux objectifs stratégiques visés par la création de la coentreprise, ou reliées aux secteurs d'activité. En intégrant dans une prochaine étude les aspects de processus de gestion avec des aspects plus structurels et stratégiques (p. ex., degré de concentration du secteur, objectif d'autonomisation de la coentreprise contre objectif de maintien de la présence québécoise), nous pourrions alors mieux comprendre le phénomène des coentreprises.

Finalement, en ce qui concerne les applications managériales de nos résultats, il faut reconnaître que nous sommes encore loin de pouvoir répondre de façon définitive aux questions que les praticiens se posent quant à la gestion des coentreprises, particulièrement dans les pays en développement. Même si les résultats de notre étude tendent à confirmer la relation entre, d'une part, le partage des informations et du contrôle managérial et, d'autre part, une performance de la coentreprise jugée satisfaisante, il faut garder en tête le caractère unique de chaque coentreprise. Il est possible que, dans certains cas, un mode de contrôle dominant soit indiqué. Cependant, il semble préférable, d'après les travaux de Beamish et à la lumière des résultats de la présente étude, que de façon générale chacun des partenaires puisse collaborer au processus de partage d'information et de contrôle, même si, à première vue, un mode de contrôle dominant peut sembler plus facile à appliquer.

Les aspects humains et socioculturels inhérents au partage d'information et au contrôle managérial doivent également être pris en considération par chacun des partenaires. Sans une bonne perception de ces facteurs, les 
partenaires risquent d'avoir beaucoup de difficultés à diminuer les coûts de transaction dans un laps de temps raisonnable, comme cela a d'ailleurs été le cas dans certaines coentreprises étudiées plus haut. Dans les cas où les partenaires des deux pays ont été rencontrés, il nous a semblé que les partenaires des pays en développement étaient plus réticents à aborder ces questions. Quelques partenaires québécois nous ont confirmé ces réticences de leurs partenaires à parler de différences culturelles; le terme lui-même « différences culturelles » semble avoir une connotation négative à leurs yeux. L'apport des conseillers privés ou gouvernementaux est important à ce chapitre.

La sélection des gestionnaires québécois affectés aux coentreprises devrait être faite autant sur des critères de compétences professionnelle ou technique que sur des critères d'aptitudes à composer avec des cultures différentes, ainsi que sur leur capacité intrapreneuriale. Le rôle de ces gestionnaires lors des premières années d'implantation est très important. Les partenaires étrangers semblent évaluer ces gestionnaires autant selon des critères techniques que socio-psychologiques et culturels.

Chaque partenaire doit s'assurer qu'il a un pouvoir de négociation suffisamment fort auprès de l'autre partenaire. Le pouvoir de négociation peut être basé sur la possession de technologie plus avancée et attirante pour le partenaire (cas $n^{\circ} 3$ ). Il peut être augmenté aussi par l'établissement de contacts avec des membres d'un réseau ou d'un groupe d'affaires auquel appartient le partenaire (cas $n^{\text {os }} 3$ et 4 ). Un autre exemple d'incitation à la collaboration par l'augmentation du pouvoir de négociation est fourni par le cas $n^{\circ} 7$. La PME québécoise impliquée dans cette coentreprise s'assure de la collaboration de son partenaire français en Afrique en lui laissant savoir que cette collaboration est nécessaire au maintien de liens d'affaires entre les deux firmes au Québec (la PME québécoise distribue les livres du partenaire français au Québec). La collaboration est un processus qui doit être constamment maintenu par des incitatifs, qu'ils soient internes ou externes à la coentreprise.

\section{Bibliographie}

Austin, J.E. (1990), Managing in Developing Countries, New York, Free Press.

BEAMISH, P.W. (1984), «Joint venture performance in developing countries », Thèse de doctorat non publiée, Université Western Ontario.

BeAmish, P.W. (1988), Multinational Joint Ventures in Developing Countries, New York, Routledge.

BEAMISH, P.W. et J.C. BANKS (1987), «Equity joint ventures and the theory of the multinational enterprise », Journal of International Business Studies, vol. 18, $n^{\circ} 2$, p. 1-16. 
Contractor, F.J. et P. Lorange (éd.) (1988), Cooperative Strategies in International Business, Toronto, Lexington Books.

Delalande, P. (1989), « Réalisation de PMI conjointes en Afrique », dans N. Lacasse et L. Perret, La coentreprise à l'étranger, Ottawa, Presses de l'Université d'Ottawa.

El Alami, A.E. (1987), «Rôle de la petite et moyenne entreprise dans le développement et la création de l'emploi : le cas de l'Afrique», Revue PMO, vol. 3, $\mathrm{n}^{\circ} 1$, p. 51-63.

GALES, L. et R.S. BLACKBURN (1990), «An analysis of the impact of supplier strategies and relationships on small retailer actions, perceptions, and performance», Entrepreneurship: Theory and Practice, automne, p. 7-21.

GERINGER, J.M. et L. HEBERT (1989), «Control and performance of international joint ventures », Journal of International Business Studies, vol. 20, nº 2, p. 235-256.

GERINGER, J.M. et L. HEBERT (1991), « Measuring performance of international joint ventures », Journal of International Business Studies, vol. 22, nº 2, p. 249-263.

GuÉNEAU, M.C. (1986), Afrique: les petits projets de développement sont-ils efficaces? Paris, Éditions L'Harmattan.

HARRIS, P.R. et R.T. MORAN (1987), Managing Cultural Differences, Houston, Gulf Publishers.

HENNART, J.F. (1988), «A transaction costs theory of equity joint ventures», Strategic Management Journal, vol. 9, nº 1, p. 361-374.

Herbolzheimer, E et H. OUANE (1985), «The transfer of technology to developing countries by small and medium sized enterprises of developed countries », dans Trade and Development: Un UNCTAD Review, New York, Nations Unies.

Julien, P.A. et M. MARCheSNAY (éd.) (1988), La petite entreprise. Principes d'économie et de gestion, Paris, Vuibert.

LARSON, A.L. (1990), «Partner networks: leveraging external ties to improve entrepreneurial performance», Frontiers of Entrepreneurship Research, Wellesley, MA, Babson College, p. 539-553.

MORRIS, D. et M.M. Hergert (1987), «Trends in international collaboration agreements », Columbia Journal of World Business, vol. 22, $\mathrm{n}^{\circ} \mathrm{2}$, p. 15-22.

SHAN, W. (1990), «An empirical analysis of organizational strategies by entrepreneurial high-technology firms », Strategic Management Journal, vol. 11, $n^{\circ} 2$, p. 129-139.

VERNON, R. et L.T. WELLS (1986), Manager in the International Economy, Englewood Cliffs, N.J., Prentice-Hall. 
Williamson, O.E. (1981), «The economics of organization : the transaction cost approach », American Journal of Sociology, vol. 87, $\mathrm{n}^{\circ} 3$, p. 548-577.

Williamson, O.E. (1985), The Economic Institutions of Capitalism, New York, Free Press.

YIN, R.K. (1984), Case Study Research, Beverly Hills, Sage Publishing. 